\title{
Robust Assessment on the Developments of Three Extended Exponential Models with Some New Properties and Applications
}

\author{
Abdullah Ali H. Ahmadini ${ }^{D},{ }^{1}$ Muhammad H. Tahir $\left(\mathbb{D},{ }^{2}\right.$ Osama Alamri $\left(\mathbb{D},{ }^{3}\right.$ \\ Alia Munawar ${ }^{2},{ }^{2,4}$ and Mohammed Elgarhy ${ }^{5}$ \\ ${ }^{1}$ Department of Mathematics, Faculty of Science, Jazan University, Jazan, Saudi Arabia \\ ${ }^{2}$ Department of Statistics, The Islamia University of Bahawalpur, Bahawalpur 63100, Pakistan \\ ${ }^{3}$ Department of Statistics, University of Tabuk, Tabuk, Saudi Arabia \\ ${ }^{4}$ Faculty of Computing, The Islamia University of Bahawalpur, Bahawalpur 63100, Pakistan \\ ${ }^{5}$ The Higher Institute of Commercial Sciences, Al-Mahalla Al Kubra, Algarbia 31951, Egypt \\ Correspondence should be addressed to Muhammad H. Tahir; mtahir.stat@gmail.com
}

Received 24 June 2021; Accepted 13 September 2021; Published 16 October 2021

Academic Editor: Ishfaq Ahmad

Copyright (c) 2021 Abdullah Ali H. Ahmadini et al. This is an open access article distributed under the Creative Commons Attribution License, which permits unrestricted use, distribution, and reproduction in any medium, provided the original work is properly cited.

\begin{abstract}
The extended exponential distribution introduced by Nadarajah and Haghighi in the year 2011, which is nowadays well known as NH distribution, has received increased attention in these days. In this paper, we provide a robust assessment report on the development of three more related extended versions of exponential (or Weibull) distributions. We assessed that which model was published earlier than the other ones and why the pioneer work was not cited properly or overlooked. For example, power generalized Weibull (PGW) introduced by Bagdonavicios and Nikulin (and Nikulin and Haghighi) and extended Weibull by Dimitrakopoulou, Adamidis, and Loukas, which we call here as the DAL model, were published earlier than the NH model. The developments of these three models are stated. A new method for the construction of NH and DAL models is outlined. A literature review on NH and DAL models is presented, and generalized classes from these models are discussed. Furthermore, some corrections in NH moments are suggested, and alternative expressions for moments and incomplete moments of $\mathrm{NH}$ and DAL models are also developed.
\end{abstract}

\section{Introduction}

In statistics and probability, one of the ever active and fragile activities is to propose mathematical models having short names such as distributions, models, and/or probability models. This activity is novel and is based on motivations and logical reasoning; see, for example, Pearson, Pareto, Burr, Johnson, and Tukey families and many more models and families of distributions published in the literature. Furthermore, many notes and letters to editors had been published in many peer-reviewed statistical and mathematical journals pointing out typos and mistakes in articles related to the model and its properties and other related issues. See, for example, some selected references [1-4]:

(i) Nadarajah [5] developed only explicit expressions for the moments of modified Weibull which was actually proposed by Xie et al. [6] as the XTG model.

(ii) Nadarajah and Kotz [7] stated that some of newly proposed modified Weibull models in the literature were not new but rather can arise from Gurvich et al. [8] generalized family.

(iii) Nadarajah and Kotz [9], the model proposed by $\mathrm{Wu}$ et al. [10], which exhibits a bathtub-shaped hazard rate, is in fact not new but originally due to Chen [11]. Furthermore, the model arises from Gurvich et al. [8] generalized family.

(iv) Bidram [12] pointed out that the proposed model "complementary exponential geometric" is not new.

(v) Lee and Tsai [13] found several typos in mathematical equations and in the incorrect proof of 
lemma in the "generalized linear exponential distribution" actually proposed by Mahmoud and Alam [14], and then the authors developed the corrected ones.

(vi) Okorie and Akpanta [15] revisited the data application and discussed the inadequacy of the model "transmuted generalized inverted exponential distribution" (TGIED) developed by Elbatal [16] and empirically investigated by Khan [17]. The authors suggested that TGIED is not a good model to study survival time data of 50 devices reported by Aarset [18], which was applied by Khan [17].

(vii) Nadarajah and Okorie [19] reported a very minor correction in the likelihood function of the "Gumbel-Burr XII model" introduced by Osatohanmwen et al. [20] and then suggested the corrected version.

(viii) Nadarajah and Zhang [21] showed that a one-parameter model can perform better as compared to the three-parameter model "transmuted inverse Weibull distribution" by Khan et al. [22] if applied to the datasets used by these authors.

(ix) Nadarajah and Chan [23] stated that the moments' and incomplete moments' expressions developed by Mazucheli et al. [24] in their developed model "one-parameter unit-Lindley distribution" are either incorrect or not in the closed form. Then, authors proposed closed-form expressions for moments and incomplete moments of the oneparameter unit-Lindley distribution.

We may find such short communications as a part of improvement(s), additional work, additional properties(s), more characterization, correction(s) in the properties, justification of the methodology or citations and criticism etc. starting with "A note on ...", "A comment on ...", "A short note on the ..., "On the moments ...", "On the distribution of ...", "On the alternative to ...", "Comment(s) on ...", "Correspondence: Letter to the Editor" etc. But our main and specific concern here and will remain always be "why some old models were published as new one without citation."

The exponential, gamma, Weibull, Lomax, Burr, and log-logistic models are basic motivating models for researchers and practitioners to think about extensions and modifications. If $T$ is a lifetime random variable, then the cumulative distribution functions (cdfs) and probability density functions (pdfs) of exponential, gamma, Weibull, Lomax, Burr, and log-logistic models are, respectively, given by

$$
\begin{aligned}
& F_{E}(t)=1-\exp \{-\mu t\} \text { and } f_{E}(t)=\mu \exp \{-\mu t\}, \quad t>0, \mu>0, \\
& F_{G a}(t)=\frac{\gamma(\delta, \lambda t)}{\Gamma(\delta)} \text { and } f_{G a}(t)=\Gamma(\delta)^{-1} \lambda^{\delta} t^{\delta-1} \exp \{-\lambda t\}, \quad t>0, \gamma, \delta>0, \\
& F_{W}(t)=1-\exp \left\{-\lambda t^{\beta}\right\} \text { and } f_{W}(t)=\lambda \beta t^{\beta-1} \exp \left\{-\lambda t^{\beta}\right\}, \quad t>0, \lambda, \beta>0, \\
& F_{L x}(t)=1-(1+\lambda t)^{-\alpha} \text { and } f_{L x}(t)=\lambda \alpha(1+\lambda t)^{-\alpha-1}, \quad t>0, \lambda, \alpha>0, \\
& F_{B r}(t)=1-\left(1+\lambda t^{\beta}\right)^{-\alpha} \text { and } f_{B r}(t)=\lambda \alpha \beta t^{\beta-1}\left(1+\lambda t^{\beta}\right)^{-\alpha-1}, \quad t>0, \lambda, \alpha, \beta>0, \\
& F_{L L}(t)=1-\left(1+\lambda t^{\beta}\right)^{-1} \text { and } f_{L L}(t)=\lambda \beta t^{\beta-1}\left(1+\lambda t^{\beta}\right)^{-2}, \quad t>0, \lambda, \beta>0,
\end{aligned}
$$

where $\gamma(p, q x)=q^{p} \int_{0}^{x} x^{p-1} \exp \{-q x\} d x$ and $\Gamma p=q^{p}$ $\int_{0}^{\infty} x^{p-1} \exp \{-q x\} d x$ are lower incomplete gamma and complete gamma functions, respectively.

In the recent past, many authors have proposed extensions and generalizations of the exponential model to increase its flexibility by adding parameters or modifying the functional form. Some extensions of exponential distributions other than Nadarajah-Haghighi (NH) (Nadarajah and
Haghighi [1]) are linear exponential (LE) (or linear failure rate) (Bain [25]), generalized exponential (GE) (Gupta and Kundu [26]), extended exponential of type 1 (ExtE1) (Mirhossaini and Dolati [27]), extended exponential of type 2 (ExtE2) (Çelebioğlu [28]), extended exponential of type 3 (ExtE3) (Olapade [29]), and extended exponential of type 4 (ExtE4) (Gómez et al. [30]). The cdfs of LE, GE, ExtE1, ExtE2, ExtE3, and ExtE4 distributions are, respectively, given by 


$$
\begin{aligned}
F_{L F R}(t) & =1-e^{-\left(\lambda t+(1 / 2) \mu t^{2}\right)}, \quad t>0, \\
F_{G E}(t) & =(1-\exp \{-\lambda t\})^{\alpha}, \quad t>0, \\
F_{E x t E 1}(t) & =(1-\exp \{\lambda t\})(1+\omega \exp \{\lambda t\}), \quad t>0, \\
F_{E x t E 2}(t) & =1-\exp \left\{\mu e^{\lambda t}-\mu t-\delta\right\}, \quad t>0, \\
F_{E x t E 3}(t) & =\frac{1}{\left[\mu^{\theta}-(\mu-1)^{\theta}\right]}\left\{[\mu-\exp (-\lambda t)]^{\theta}-(\mu-1)^{\theta}\right\}, \quad t>0, \\
F_{E x t E 4}(t) & =\frac{\lambda+\mu-(\lambda+\mu-\lambda \mu t) \exp \{-\lambda t\}}{\lambda+\mu}, \quad t>0,
\end{aligned}
$$

where $\lambda>0, \mu>0, \delta>0$, and $-1<\omega<1$ are scale parameters, while $\theta>0$ and $\alpha>0$ are power (or shape) parameters. This paper is organized as follows: the main concern regarding the actual proposal of the extended exponential model is addressed in Section 2. A useful development procedure for Nadarajah-Haghighi and Dimitrakopoulou, Adamidis, and Loukas models is described in Section 3. A brief review of the literature on Nadarajah-Haghighi and Dimitrakopoulou, Adamidis, and Loukas models is given in Section 4. In Section 5, some corrections in the moments of the Nadarajah-Haghighi model and an alternate method for the moments of Nadarajah-Haghighi and Dimitrakopoulou, Adamidis, and Loukas are developed. The final Section 6 concludes the paper listing further G-classes that can be developed from Nadarajah-Haghighi and Dimitrakopoulou, Adamidis, and Loukas models.

\section{The Main Objective of Research}

In this section, we consider the three related models, namely, Nadarajah-Haghighi, power generalized Weibull, and Dimitrakopoulou, Adamidis, and Loukas distributions, and then discuss the development of these models to prove "to whom the credit actually must go naturally as the pioneer."

Bagdonavicios and Nikulin ([2], p. 110) first introduced a generalized Weibull family which exhibits all possible hazard rate shapes constant, increasing and decreasing (monotone), and bathtub and upside-down bathtub (nonmonotone) and later called it as the generalized power Weibull (GPW) and power generalized Weibull (PGW) distribution by Nikulin and Haghighi [31,32], respectively. The GW and GPW are nowadays popular as the PGW model, which is actually an extension of exponential, Weibull, and extended exponential (NH) models. The cdf of PGW (due to Bagdonavicios and Nikulin [2] and Nikulin and Haghighi $[31,32]$ ) is given by

$$
F_{P G W}(x)=1-\exp \left\{1-\left[1+\left(\frac{x}{\lambda}\right)^{\beta}\right]^{(1 / \gamma)}\right\}, \quad x>0,
$$

where $\lambda>0$ is the scale parameter, while $\gamma>0$ and $\beta>0$ are shape parameters.

Dimitrakopoulou, Adamidis, and Loukas [4] (which we acronym here as DAL based on the last names of the authors) proposed another extension of the exponential and/or Weibull distribution. The cdf and pdf of the DAL distribution are, respectively, given by

$$
\begin{aligned}
& F_{D A L}(x)=1-\exp \left\{1-\left(1+\lambda x^{\beta}\right)^{\alpha}\right\}, \quad x>0, \\
& f_{D A L}(x)=\lambda \alpha \beta x^{\beta-1}\left(1+\lambda x^{\beta}\right)^{\alpha-1} \exp \left\{1-\left(1+\lambda x^{\beta}\right)^{\alpha}\right\},
\end{aligned}
$$

where $\lambda>0$ is the scale parameter, while $\alpha>0$ and $\beta>0$ are shape parameters.

Note 1. Although there seems some difference in the cdfs of DAL and PGW, in general (after reparametrization), both models are similar (see Peña-Ramírez et al. [33]).

Nadarajah and Haghighi [1] introduced the extended version of the exponential distribution and called it extended exponential, which is well known as the Nadarajah-Haghighi $(\mathrm{NH})$ distribution. The cdf and pdf of the $\mathrm{NH}$ model are, respectively, given by

$$
\begin{aligned}
& F_{N H}(x)=1-\exp \left\{1-(1+\lambda x)^{\alpha}\right\}, \quad x>0, \\
& f_{N H}(x)=\lambda \alpha(1+\lambda x)^{\alpha-1} \exp \left\{1-(1+\lambda x)^{\alpha}\right\}, \quad x>0,
\end{aligned}
$$

where $\lambda>0$ is the scale parameter, while $\alpha>0$ is the shape parameter.

Note 2. There is one special case of the NH model, that is, the exponential distribution if $\alpha=1$, but there are three special cases of the PGW or DAL model: (i) if $\beta=1$, the PGW or DAL reduces to the NH distribution, (ii) if $\alpha=1$, PGW or DAL reduces to the Weibull distribution, and (iii) if $\alpha=\beta=1$, PGW or DAL reduces to the exponential distribution. Furthermore, more special cases can be generated with the help of variable transformations (see, for example, Dimitrakopoulou et al. [4], pp. 308-9). 
2.1. Comparing Developments of PGW vs. DAL vs. NH Models. The PGW appeared first in the book of Bagdonavicios and Nikulin ([2], p. 110) under the title "Accelerated Life Models" published by a well-known publisher, the Chapman \& Hall/ CRC, London, which may be read by every statistician and researcher interested in distribution theory, reliability analysis, and lifetime modelling. Furthermore, books or monographs of Chapman \& Hall/CRC and Wiley publishers are available in most of libraries of every country and are in easy access of the students, teachers, and practitioners. The idea of flexible hazard rate (all possible hazard rate shapes) was reconsidered in an article by Nikulin and Haghighi [31] while proposing a $\chi^{2}$-test for the PGW family, and then the PGW model was empirically investigated in the presence of type-II censoring for "Head and Neck Cancer Data." It is evident that Mr. Nikulin has expertise in $\chi^{2}$-testing and had coauthored a book with P. Greenwood under Wiley publisher titled "A Guide to Chi-square Testing."

Detail properties of PGW such as quantile, analytical shapes of the density and hazard rate, moments, mode, parameter estimation by the maximum likelihood method, simulation study, and empirical investigation through "Head and Neck Cancer Data" were studied by Nikulin and Haghighi [32].

The DAL model (same as PGW) was published in 2007 by Dimitrakopoulou, Adamidis, and Loukas (which they actually submitted in the IEEE journal in 2006) in which they took the same plea on proposing a model with flexible hazard rate shapes, stated relations with submodels (by using transformations), presented motivation in the risk scenario, and investigated the model properties such as quantile function, moments, hazard rate behaviour, and parameter estimation, but did not cite the three earlier works.

The NH model which appeared online in 2010 (16 March 2010) and was published in 2011 did not consider citing the previous four key references despite the fact that one of the coauthors was well aware of the development of PGW (a more extended model than $\mathrm{NH}$ ). It was better if the authors of the NH model had given a credit to deserving ones.

Evidently, the three articles on PGW (2009), DAL (2007), and $\mathrm{NH}$ (2011) are useful extensions of the exponential or Weibull models and apparently look similar up to some extent (model formulation) but differ with respect to the type and number of parameters, motivation, content, and presentation.

Finally, after thorough consideration, we may be able to conclude that the model proposed by Nadarajah and Haghighi [1] was not new but in fact a special case of the PGW or DAL model as an extended Weibull model or extended exponential model pioneered by Bagdonavicios and Nikulin [2] and Nikulin and Haghighi [31] and in some way Dimitrakopoulou et al. [4]. We find it very difficult to admit that the four references related to PGW or DAL models were slipped from the attention of NH model's authors.

\section{A Useful Development Procedure of NH, PGW, or DAL Models}

If $G(x)$ and $\bar{G}(x)=1-G(x)$ are the cdf and survival function (sf) of the baseline model, then odd ratio is defined as $G(x) / \bar{G}(x)$. Following the T-X criterion (Alzaatreh et al. [34]), the cdf of the odd exponential-G (OEG) class is defined by

$$
F_{O E G}(x)=\int_{0}^{G(x) / \bar{G}(x)} \mu \mathrm{e}^{-\mu t} d t=1-\exp \left\{-\mu\left[\frac{G(x)}{\bar{G}(x)}\right]\right\} .
$$

Many new composite models can be generated from the OEG class. Table 1 lists some baseline models, their odd ratios $G(x) / \bar{G}(x)$, and published models of some wellknown distributions generated from the OEG class.

\section{Literature Review on NH and DAL Models}

In this section, we present a needful review to $\mathrm{NH}$, exponentiated $\mathrm{NH}$, inverted $\mathrm{NH}$, and PGW (or DAL) models. Details on extended or generalized $\mathrm{NH}$ and PGW (or DAL) models are out of scope of this article, and the interested readers may read the referred (or cited) articles directly.

4.1. NH Model. Nadarajah and Haghighi [1] proposed three motivations for the $\mathrm{NH}$ model, reported some useful mathematical properties such as quantile, analytical shapes of the density and hazard rate, moments (complete and incomplete), L-moments, Bonferroni and Lorenz curves, Rényi entropy, and order statistics, and also dealt parameter estimation. The $\mathrm{NH}$ density offers reversed-J and rightshewed shapes, while the hazard rate shapes could be increasing, decreasing, and constant (not very attractive from the plotted graphs).

4.1.1. Order Statistics and Records. Kumar et al. [36] established recurrence relations for the single and product moments of order statistics from the $\mathrm{NH}$ distribution. MirMostafaee et al. [37] obtained some recurrence relations for the single and product moments of upper records from the NH model. Selim [38] dealt estimation of $\mathrm{NH}$ model parameters through the maximum likelihood and Bayes method based on record values and also considered point and interval predictions of the future record values. Khan and Sharma [39] obtained exact expressions for the Shannon entropy of the NH model based on generalized order statistics. Sana and Faizan [40] considered maximum likelihood and Bayesian estimation of the $\mathrm{NH}$ model based on upper records and obtained Bayes estimates under squared error loss, balanced squared error, and general entropy loss functions.

\subsubsection{Life Testing under Censoring Schemes. Haghighi [41]} introduced a simple step-stress accelerated life test and derived an optimum plan for the NH model. Haghighi [42] proposed a design for the step-stress accelerated life test for the $\mathrm{NH}$ distribution in the presence of type-I censoring and then estimated model parameters for such circumstances. El-Din et al. [43] also proposed a simple step-stress accelerated life test for the $\mathrm{NH}$ model under type-II progressive censoring, obtained maximum likelihood and Bayes 
TABLE 1: Some special models of the OE G-class of distributions.

\begin{tabular}{lcccc}
\hline Distribution & $G(x)$ & $G(x) / \bar{G}(x)$ & Generated cdf & The model \\
\hline Weibull & $1-\mathrm{e}^{-x^{\beta}}$ & $\mathrm{e}^{x^{\beta}}-1$ & $1-\exp \left[\mu\left(1-\mathrm{e}^{x^{\beta}}\right)\right]$ & Chen [11] \\
Weibull & $1-\mathrm{e}^{-(x / \sigma)^{\beta}}$ & $\mathrm{e}^{(x / \sigma)^{\beta}}-1$ & $1-\exp \left[\mu\left(1-\mathrm{e}^{(x / \sigma)^{\beta}}\right)\right]$ & Xie et al. [6] \\
Lomax & $1-(1+\lambda x)^{-\alpha}$ & $(1+\lambda x)^{\alpha}-1$ & $1-\exp \left[1-(1+\lambda x)^{\alpha}\right]($ when $\mu=1)$ & NH \\
Burr XII & $1-\left(1+\lambda x^{\beta}\right)^{-\alpha}$ & $\left(1+\lambda x^{\beta}\right)^{\alpha}-1$ & $1-\exp \left[1-\left(1+\lambda x^{\beta}\right)^{\alpha}\right]($ when $\mu=1)$ & PGW and DAL \\
Exponential & $1-\mathrm{e}^{-\mu x}$ & $\mathrm{e}^{\mu x}-1$ & $1-\exp \left[-(\sigma / \mu)\left(\mathrm{e}^{\mu x}-1\right)\right]($ when $\mu=\sigma / \mu)$ & Gompertz $[35]$ \\
\hline
\end{tabular}

estimates for $\mathrm{NH}$ model parameters, and also derived approximate, bootstrap, and credible intervals for the estimators. El-Din et al. [44] considered the progressive stress accelerated life test under progressive type-II censoring and obtained parameter estimates of the $\mathrm{NH}$ model through maximum likelihood and Bayes methods of estimation along with Bayes credible intervals. Singh et al. [45] considered parameter estimation using classical and Bayesian methods for $\mathrm{NH}$ model parameters under progressive type-II censoring under binomial removal. El-Raheem [46] considered the optimal allocation problem in multiple constant-stress accelerated life testing for the $\mathrm{NH}$ model under type-II censoring.

4.1.3. Discrete NH Versions. Kumar et al. [47] proposed a discrete version of the $\mathrm{NH}$ model, which they called count extended exponential model $\left(C_{n}(t)\right)$ using the following formula: $C_{n}(t)=F_{n}(t)-F_{n+1}(t), \quad n=0,1, \ldots$, and investigated some mathematical properties. Recently, Ali et al. [48] suggested the bivariate discrete NH model and reported some useful mathematical properties along with the estimation of model parameters with the help of seven wellknown methods.

4.1.4. T-X Family for the NH Model. Recently, Nasiru et al. [49] proposed the $\mathrm{T}-\mathrm{NH}\{\mathrm{Y}\}$ family based on the quantile function approach pioneered by Alzaatreh et al. [34] and Aljarrah et al. [50] having cdf

$$
\begin{aligned}
F_{X}(x) & =\int_{0}^{1-\exp \left\{1-(1+\lambda x)^{\alpha}\right\}} f_{T}(t) \mathrm{d} t \\
& =F_{T}\left(Q_{Y}\left[1-\exp \left\{1-(1+\lambda x)^{\alpha}\right\}\right]\right), \quad x, \theta, \lambda>0,
\end{aligned}
$$

where $F_{R}(x)=\left[1-\exp \left\{1-(1+\lambda x)^{\alpha}\right\}\right]$. Nasiru et al. [49] also investigated mathematical properties of the T-NH$\{\mathrm{Y}\}$ family such as mode, quantiles, moments, and Shannon entropy along with the estimation of parameters, simulation study, and empirical investigation.

4.1.5. Truncated (Unit) NH Version. Recently, Nasiru et al. [51] developed the truncated version of the $\mathrm{NH}$ model for bounded unit interval $(0,1)$ based on the left truncation criterion and then proposed the unit Nadarajah-Haghighi (UNH) model and unit Nadarajah-Haghighi generalized (UNH-G) family having cdf, respectively, as follows:

$$
\begin{gathered}
F_{U N H}(x)=\frac{1-\exp \left\{1-(1+\lambda x)^{\alpha}\right\}}{1-\exp \left\{1-(1+\lambda)^{\alpha}\right\}}, \quad x \in(0,1), \lambda, \alpha>0, \\
F_{U N H G}(x)=\frac{1-\exp \left\{1-(1+\lambda G(x ; \xi))^{\alpha}\right\}}{1-\exp \left\{1-(1+\lambda)^{\alpha}\right\}}, \quad x, \lambda, \alpha>0 .
\end{gathered}
$$

They investigated some useful properties of the UNH-G family and performed simulation studies for the two special models UNH-Weibull and UNH-log-logistic along with empirical investigation. It is pertinent to mention here that recently, Alzaatreh et al. [52] proposed and studied righttruncated and left-truncated T-X families of distributions, a more generalized concept and formulation.

4.1.6. Methods of Estimation. Singh et al. [53] considered the classical and Bayesian estimation of the $\mathrm{NH}$ model parameters and reliability characteristics. Dey et al. [54] investigated the estimation of $\mathrm{NH}$ model parameters by using methods of the maximum likelihood, moment, percentile, least squares and weighted least squares, and Bayesian and compared them using a simulation study. Minić [55] estimated $\mathrm{NH}$ model parameters using simple random sampling following the maximum likelihood method, moment method, and modified maximum likelihood method and also using ranked set sampling under imperfect and perfect ranking. Through simulation study, Minic showed that the estimators obtained through ranked set sampling using perfect or imperfect ranking are better in performance as compared to estimators obtained through simple random sampling.

4.1.7. Miscellaneous Contributions to the NH Model. El-Damcese and Ramadan [56] proposed and studied the modified version of $\mathrm{NH}$, called modified Nadarajah-Haghighi, having cdf

$$
F_{M N H}(x)=1-\exp \left\{1-\left(1+\lambda x+\delta x^{2}\right)^{\alpha}\right\}, \quad x, \lambda, \alpha, \delta>0,
$$

where $\lambda$ and $\delta$ are scale parameters, while $\alpha$ is the shape parameter.

Khan et al. [57] introduced a weighted version of the $\mathrm{NH}$ model by defining the cdf as 


$$
F_{W N H}(x)=\frac{1-\exp \left\{1-(1+\lambda x)^{\alpha}\right\}}{1+\exp \left\{1-(1+\lambda x)^{\alpha}\right\}}, \quad x, \lambda, \alpha>0,
$$

and studied a very few basic properties.

Peña-Ramírez et al. [58] proposed a compounded NHLindley model for components of a system arranged in series, having a new survival function (product of two sfs) as

$$
\begin{array}{r}
F_{N H L}(x)=\frac{1+\theta+\theta x}{1+\theta} \exp \{-\theta x\} \exp \left\{1-(1+\lambda x)^{\alpha}\right\}, \\
x, \theta, \lambda>0 .
\end{array}
$$

4.2. Exponentiated NH Model. Lemonte [59] proposed and studied a simple extension of $\mathrm{NH}$ by using Lehmann alternative 1 (see Gupta et al. [60]) called exponentiated $\mathrm{NH}$ (ENH) distribution. The induction of one additional shape parameter into $\mathrm{NH}(\mathrm{ENH})$ resulted in flexible shapes of the density and hazard rate. The ENH density can exhibit reversed-J, symmetrical, and right-shewed, while the hazard rate shapes are increasing, decreasing, bathtub, and upsidedown bathtub. Lemonte obtained useful mathematical properties such as quantile function, analytical shapes of the density and hazard rate, moments, MacGillivray's skewness measure, entropies and Kullback-Leibler divergence, stressstrength reliability parameter, and estimation of model parameters. Sira et al. [61] proposed a compound version of ENH called ENH-Poisson model and investigated some mathematical properties. Recently, Saboor et al. [62] developed an extended ENH model called beta-ENH which offers flexible shapes of the density and hazard rate. Alhussain and Ahmed [63] considered classical and Bayesian methods of the estimation of ENH model parameters under progressive type-II censoring.

4.3. Inverted NH (INH) Model. Tahir et al. [64] introduced the inverted version of the $\mathrm{NH}$ model, obtained some mathematical properties, and compared INH model parameters through a simulation study by using different methods of estimation such as maximum likelihood, least squares and weighted least squares, maximum product spacing, Cramér-von Mises, Anderson-Darling, right-tail Anderson-Darling, and Bayesian. Recently, Raffiq et al. [65] proposed the Marshall-Olkin INH distribution and obtained a very few basic mathematical properties.

4.4. PGW (or DAL) Model. Bagdonavicios and Nikulin ([2], p. 110) first proposed the PGW model as the family of scaleshape distributions, as an alternate to the Weibull, log-logistic, and lognormal distribution, which exhibits all possible shapes hazard rate. The cdf is given by $F(x)=1-\exp \left\{1-\left[1+(x / \lambda)^{\beta}\right]^{\alpha}\right\}, \quad x, \lambda, \alpha, \beta>0$. Nikulin and Haghighi [31] while discussing Weibull, exponentiated Weibull (Mudholkar and Srivastava [66]), and generalized Weibull (Mudholkar et al. [67]) families of distributions which exhibit monotone hazard rates described the PGW family along with empirical investigation to censored data and clearly stated in page 1336 that Bagdonavicios and Nikulin [2] were the first who introduced the GPW family of distributions. Dimitrakopoulou, Adamidis, and Loukas [4] introduced the GPW model claiming flexible hazard rate shapes, motivated the model in the risk scenario, and obtained some mathematical properties such as quantile function, moments, and hazard rate behaviour along with parameter estimation. Nikulin and Haghighi [32] obtained mathematical properties of PGW such as quantile, analytical shapes of the density and hazard rate, moments, and mode along with parameter estimation by the maximum likelihood method, simulation study, and empirical investigation.

Voinov et al. [68] proposed modified goodness-of-fit tests based on the maximum likelihood of PGW parameters and, through Monte Carlo simulation, showed that power of the tests of the PGW model (cited reference of Bagdonavicios and Nikulin [2]) is better than two-parameter Weibull, three-parameter Weibull, and generalized Weibull models. Kumar and Dey [69] developed the recurrence relation for the single and product moments of order statistics from the PGW model and stated that this model is actually due to Bagdonavičios and Nikulin [2]. Kumar and Jain [70] obtained explicit expressions for the recurrence relation for the single, product, and conditional moments of order statistics from the PGW model and stated that it is due to Bagdonavicios and Nikulin [2]. Pandey and Kumari [71] used the Bayesian estimation approach for the parameter estimation of GPW while considering Lindley's approximation and Markov chain Monte Carlo under type-II censoring. Sabry et al. [72] used double-ranked set sampling (DRSS) and general doubleranked set sampling (GDRSS) approaches for the parameter estimation of the GPW model and proved through simulation study that the GDRSS approach yields more efficient results as compared to ranked set sampling, extreme ranked set sampling, and DRSS schemes. Almetwaly and Almongy [73] dealt parameters' estimation of the GPW model through classical and Bayesian methods for the complete sample and censored samples (type-II censoring and type-II progressive censoring schemes). El-Din et al. [74] considered step-stress accelerated life testing for testing the lifetime of GPW and used maximum likelihood and Bayesian methods for the estimation of model parameters under type-II progressive censoring. Jones et al. [75] developed the bivariate version of GPW, defined its copula presentation, and then investigated bivariate shared frailty of adaptive PGW and bivariate shared frailty of PGW models.

4.5. Extended NH Model from Other G-Classes. Some generalizations of the $\mathrm{NH}$ model were reported in the literature which are listed in Table 2.

4.6. Extended PGW (or DAL) Model from Other G-Classes. Some generalizations of the PGW (or DAL) model were reported in the literature which are listed in Table 3. 
TABLe 2: Some extended NH models from other G-classes.

\begin{tabular}{lccc}
\hline Distribution & Year & Authors & G-class used \\
\hline Exp-NH & 2013 & Lemonte & Exp-G (LA1) (Gupta et al. [60]) \\
Gamma NH & 2015 & Bourguignon et al. & Gamma-G (Zografos and Balakrishnan [76]) \\
Transmuted NH & 2015 & Ortega et al. & Gamma-G (Zografos and Balakrishnan [76]) \\
EG-NH & 2015 & Ahmed et al. & Transmuted-G (Shaw and Buckley [77]) \\
MONH & 2016 & VedoVatto et al. & EG-G (Cordeiro et al. [78]) \\
TL-NH & 2016 & Lemonte et al. & Marshall-Olkin-G (Marshall and Olkin [79]) \\
Odd Lindley-NH & 2017 & Yousof and Korkmaz & Topp-Leone-G (Sangsanit and Bodhisuwan [80]) \\
Odd Weibull-NH & 2017 a & Yousof et al. & Odd Lindley-G (Gomes-Silva et al. [81]) \\
Kumaraswamy-NH & $2018 \mathrm{a}$ & Peña-Ramrírez et al. & Odd Weibull-G (Bourguignon et al. [82]) \\
Beta NH & 2018 & Elbatal et al. & Kumaraswamy-G (Cordeiro and de Castro [83]) \\
Half-logistic NH & 2018 & Dias et al. & Beta-G (Eugene et al. [84]) \\
ExtExp-NH & 2018 & Anwar and Bibi & Half-logistic-G (Cordeiro et al. [85]) \\
Burr X-NH & 2018 & Alizadeh et al. & Extended exp-G (Alizadeh et al. [86]) \\
APT-NH & 2019 & Elsayed and Yousof & Burr-X-G (Yousof et al. [87]) \\
NH-Poisson & 2019 & Hassan et al. & APT-G (Mahdavi and Kundu [88]) \\
OLL-NH & 2020 & Mansoor et al. & Compounded with ZTP \\
Logistic-NH & 2020 & Ibrahim & OLLG (Gleaton and Lynch [89]) \\
\hline
\end{tabular}

TABLE 3: Some extended PGW (or DAL) models from other G-classes.

\begin{tabular}{lccc}
\hline Distribution & Year & Authors & G-class used \\
\hline Exponentiated DAL & $2018 \mathrm{~b}$ & Peña-Ramírez et al. & Exp-G (LA1) (Gupta et al. [60]) \\
Half-logistic DAL & 2018 & Anwar and Bibi & Half-logistic-G (Cordeiro et al. [85]) \\
DAL-logarithmic & 2018 & Tafakori et al. & Compounded with ZT logarithmic \\
Transmuted DAL & $2018 \mathrm{~b}$ & Khan & Transmuted-G (Shaw and Buckley [77]) \\
MO-DAL & 2020 & Afify et al. & Marshall-Olkin-G (Marshall and Olkin [79]) \\
\hline
\end{tabular}

\section{G-Classes from NH and DAL (or PGW) Models}

Alzaatreh et al. [34] proposed a general method for constructing G-classes by using the transformed-transformer (T-X) approach. Let $r(t)$ be the pdf and $R(t)$ be the cdf of a rv $T \in[a, b]$ for $-\infty<a<b<\infty$, and let $W[G(x)]$ be a function of the cdf $G(x)$ or sf $\bar{G}(x)$ of any baseline rv $(W(\cdot)$ is known as the generator) such that $W[G(x)]$ satisfies three conditions:

(i) $W[G(x)] \in[a, b]$

(ii) $W[G(x)]$ is differentiable and monotonically nondecreasing

(iii) $\lim _{x \longrightarrow-\infty} W[G(x)]=a$ and $\lim _{x \longrightarrow \infty} W[G(x)]=b$
The cdf of the $T-X$ family is

$$
F_{T X}(x)=\int_{a}^{W[G(x)]} r(t) \mathrm{d} t=R(W[G(x)]),
$$

where $W[G(x)]$ satisfies conditions (i)-(iii).

The pdf corresponding to equation (15) is

$$
f_{T X}(x)=r(W[G(x)]) \frac{d}{d x} W[G(x)] .
$$

For $T \in[0, \infty)$, the following generators $W[G(\cdot)]$ have been reported so far, which can be used to define $\mathrm{NH}$ G-classes for rv $T$ : (i) $-\log \bar{G}(x)$ (Alzaatreh et al. [34]), (ii) $G(x) / \bar{G}(x)$ (odds) (Bourguignon et al. [82]), and (iii) $[-\log \bar{G}(x)] / \bar{G}(x)$ (Ahmad et al. [91]). 


$$
\begin{gathered}
F_{N H G 1}(x)=1-\exp \left\{1-[1-\lambda \log [\bar{G}(x ; \xi)]]^{\alpha}\right\}, \quad x>0, \lambda, \alpha>0, \\
F_{N H G 2}(x)=1-\exp \left\{1-\left[1-\lambda \frac{\log [\bar{G}(x ; \xi)]}{\bar{G}(x ; \xi)}\right]^{\alpha}\right\}, \quad x>0, \lambda, \alpha>0, \\
F_{O N H G}(x)=1-\exp \left\{1-\left[1+\lambda \frac{G(x ; \xi)}{\bar{G}(x ; \xi)}\right]^{\alpha}\right\}, \quad x>0, \lambda, \alpha>0, \\
F_{N H T L-G}(x)=1-\exp \left\{1-\left[1+\lambda\left\{1-\bar{G}(x ; \xi)^{2}\right\}^{\theta}\right]^{\alpha}\right\}, \quad x>0, \lambda, \alpha, \theta>0 .
\end{gathered}
$$

For $T \in[0, \infty)$, the following generators $W[G(x)]$ have been reported so far, which can be used to define DAL G-classes for rv T: (i) $-\log \bar{G}(x)$ (Zografos and Balakrishnan
[76]; Alzaatreh et al. [34]), (ii) $G(x) / \bar{G}(x)$ (odds) (Gleaton and Lynch [89]; Bourguignon et al. [82]), and (iii) $[-\log \bar{G}(x)] / \bar{G}(x)$ (Ahmad et al. [91]).

$$
\begin{aligned}
& F_{D A L G 1}(x)=1-\exp \left\{1-\left[1+\lambda(-\log [\bar{G}(x ; \xi)])^{\beta}\right]^{\alpha}\right\}, \quad x>0, \lambda, \alpha, \beta>0, \\
& F_{D A L G 2}(x)=1-\exp \left\{1-\left[1+\lambda\left(\frac{-\log [G(x ; \xi)]}{\bar{G}(x ; \xi)}\right)^{\beta}\right]^{\alpha}\right\}, \quad x>0, \lambda, \alpha, \beta>0,
\end{aligned}
$$

and

$$
\begin{array}{r}
F_{O D A L G}(x)=1-\exp \left\{1-\left[1+\lambda\left(\frac{G(x ; \xi)}{\bar{G}(x ; \xi)}\right)^{\beta}\right]^{\alpha}\right\}, \\
x>0, \lambda, \alpha, \beta>0,
\end{array}
$$

where $\lambda>0$ is the scale parameter, while $\alpha>0$ and $\beta>0$ are shape parameters.

Note 3. The generator $[-\log G(x)]$ does not fulfil the conditions of the T-X family of distributions.

Note 4. It may be possible that the above G-classes may create nonidentifiability issue itself or after choosing some baseline models.
Nascimento et al. [92] and Reyad et al. [93] introduced and studied ONH-G and NH-TL-G classes of distributions. Ahmad et al. [94] proposed the odd DAL-G class, while Nasiru and Abubakari [95] and Aldahlan et al. [96] proposed complementary generalized power Weibull power series and exponentiated power generalized Weibull power series families of distributions.

\section{Corrected Moments of the NH Model}

Following (5), the corrected $r$ th moment expression will be

$$
\mathbb{E}\left(T^{k}\right)=\frac{e}{\lambda^{k}} \sum_{i=0}^{k}\left(\begin{array}{c}
k \\
i
\end{array}\right)(-1)^{k-i} \Gamma\left(\frac{i}{\alpha}+1,1\right),
$$

and the first four moments of $T$ are given by

$$
\begin{aligned}
\mathbb{E}(T) & =\frac{e}{\lambda}\left[-1+\Gamma\left(\frac{1}{\alpha}+1,1\right)\right] \\
\mathbb{E}\left(T^{2}\right) & =\frac{e}{\lambda^{2}}\left[1-2 \Gamma\left(\frac{1}{\alpha}+1,1\right)+\Gamma\left(\frac{2}{\alpha}+1,1\right)\right] \\
\mathbb{E}\left(T^{3}\right) & =\frac{e}{\lambda^{3}}\left[-1+3 \Gamma\left(\frac{1}{\alpha}+1,1\right)-3 \Gamma\left(\frac{2}{\alpha}+1,1\right)+\Gamma\left(\frac{3}{\alpha}+1,1\right)\right] \\
\mathbb{E}\left(T^{4}\right) & =\frac{e}{\lambda^{3}}\left[1-4 \Gamma\left(\frac{1}{\alpha}+1,1\right)+6 \Gamma\left(\frac{2}{\alpha}+1,1\right)-4 \Gamma\left(\frac{3}{\alpha}+1,1\right)+\Gamma\left(\frac{4}{\alpha}+1,1\right)\right] .
\end{aligned}
$$

The first four upper incomplete $r$ th moments given in Nadarajah and Haghighi ([1], p. 549) include one extra term $\exp \left\{(1+\lambda x)^{\alpha}\right\}$ as the common numerator. Therefore, the corrected incomplete $r$ th moment expression will be 


$$
\mathbb{E}\left(T^{k} \mid T>t\right)=\frac{e}{\lambda^{k}} \sum_{i=0}^{k}\left(\begin{array}{c}
k \\
i
\end{array}\right)(-1)^{k-i} \Gamma\left(\frac{i}{\alpha}+1, \omega\right)
$$

$$
\begin{aligned}
\mathbb{E}(T \mid T>t) & =\frac{e}{\lambda}\left[-1+\Gamma\left(\frac{1}{\alpha}+1, \omega\right)\right], \\
\mathbb{E}\left(T^{2} \mid T>t\right) & =\frac{e}{\lambda^{2}}\left[1-2 \Gamma\left(\frac{1}{\alpha}+1, \omega\right)+\Gamma\left(\frac{2}{\alpha}+1, \omega\right)\right], \\
\mathbb{E}\left(T^{3} \mid T>t\right) & =\frac{e}{\lambda^{3}}\left[-1+3 \Gamma\left(\frac{1}{\alpha}+1, \omega\right)-3 \Gamma\left(\frac{2}{\alpha}+1, \omega\right)+\Gamma\left(\frac{3}{\alpha}+1, \omega\right)\right], \\
\mathbb{E}\left(T^{4} \mid T>t\right) & =\frac{e}{\lambda^{3}}\left[1-4 \Gamma\left(\frac{1}{\alpha}+1, \omega\right)+6 \Gamma\left(\frac{2}{\alpha}+1, \omega\right)-4 \Gamma\left(\frac{3}{\alpha}+1, \omega\right)+\Gamma\left(\frac{4}{\alpha}+1, \omega\right)\right],
\end{aligned}
$$

where $\exp \left\{(1+\lambda x)^{\alpha}\right\}$. Sometimes, the lower incomplete moments are of interest for the researchers to investigate additional properties of the model. For example, the first lower incomplete moment is used to compute Bonferroni and Lorenz curves and to determine the totality of deviations from the mean and median of $X$. Therefore, the lower incomplete $r$ th moment expression is given by

$$
\mathbb{E}\left(T^{k} \mid T<t\right)=\frac{e}{\lambda^{k}} \sum_{i=0}^{k}\left(\begin{array}{c}
k \\
i
\end{array}\right)(-1)^{k-i}\left[\Gamma\left(\frac{i}{\alpha}+1,1\right)-\Gamma\left(\frac{i}{\alpha}+1, \omega\right)\right]
$$

(see https://www.wolframalpha.com), where $\gamma(p, x)=$ $\Gamma(p)^{-1} \int_{0}^{x} t^{p-1} \exp \{-t\} d t, \quad \Gamma(p, x)=\Gamma(p)^{-1} \quad \int_{x}^{\infty} t^{p-1} \exp$ $\{-t\} d t$, and $\Gamma(p)=\Gamma(p)^{-1} \int_{0}^{\infty} t^{p-1} \exp \{-t\} d t$ are lower incomplete gamma, upper incomplete gamma, and complete gamma functions, respectively.

\section{Alternate Expressions for the rth Moments of the NH Model}

Using transformation $\omega=\exp \left\{(1+\lambda x)^{\alpha}\right\}$ in (7), the (HTML translation failed)th moment expression for the $\mathrm{NH}$ model becomes

$$
\mathbb{E}\left(T^{k}\right)=\frac{e}{\lambda^{k}} \int_{1}^{\infty}(-1)^{k}\left(1-\omega^{(1 / \alpha)}\right)^{k} \exp \{-\omega\} d \omega
$$

By using the binomial expansion given by Cordeiro and Andrade [97, 98] (for $|\omega|<0$ ), we can write

$$
\left(1-y^{1 / \gamma}\right)^{r}=1+\sum_{i=1}^{\infty} \frac{(-1)^{i+1}}{i !} \prod_{j=0}^{i-1}(r-j) y^{i / \gamma}=\sum_{i=0}^{\infty} a_{i}(r) y^{i / \gamma}
$$

where $a_{i}(r)=(-1)^{i+1} / i ! \prod_{j=0}^{i-1}(r-j)$.

From the last two results, the alternative $k$ th ordinary moment expression for $\mathrm{NH}$ results in

$$
\mathbb{E}\left(T^{k}\right)=\frac{\mathrm{e}}{\lambda^{k}}(-1)^{k} \sum_{i=0}^{\infty} a_{i}(k) \int_{1}^{\infty} \omega^{(i / \alpha)} \exp \{-\omega\} d \omega=\frac{\mathrm{e}}{\lambda^{k}}(-1)^{k} \sum_{i=0}^{\infty} a_{i}(k) \Gamma\left(\frac{i}{\alpha}+1,1\right) .
$$

In a similar way, the alternate $r$ th incomplete moment expressions for $\mathrm{NH}(T>t$ and $T<t)$ can be deduced and are

$$
\mathbb{E}\left(T^{k} \mid T>t\right)=\frac{\mathrm{e}}{\lambda^{k}}(-1)^{k} \sum_{i=0}^{\infty} a_{i}(k) \int_{1}^{\infty} \omega^{(i / \alpha)} \exp \{-\omega\} d \omega=\frac{\mathrm{e}}{\lambda^{k}}(-1)^{k} \sum_{i=0}^{\infty} a_{i}(k) \Gamma\left(\frac{i}{\alpha}+1, \omega\right),
$$


and

$$
\mathbb{E}\left(T^{k} \mid T<t\right)=\frac{e}{\lambda^{k}}(-1)^{k} \sum_{i=0}^{\infty} a_{i}(k) \int_{1}^{\infty} \omega^{(i / \alpha)} \exp \{-\omega\} d \omega=\frac{e}{\lambda^{k}}(-1)^{k} \sum_{i=0}^{\infty} a_{i}(k)\left[\Gamma\left(\frac{i}{\alpha}+1,1\right)-\Gamma\left(\frac{i}{\alpha}+1, \omega\right)\right]
$$

\section{Moments of the PGW (or DAL) Model}

Following (3), th $r$ th moment expression for PGW will be

$$
\mathbb{E}\left(T^{k} \mid T>t\right)=\frac{e}{\lambda^{(k / \beta)}} \sum_{i=0}^{\infty}\left(\begin{array}{c}
(k / \beta) \\
i
\end{array}\right)(-1)^{(k / \beta-i)} \Gamma\left(\frac{i}{\alpha}+1, \emptyset\right),
$$

$$
\mathbb{E}\left(T^{k}\right)=\frac{e}{\lambda^{(k / \beta)}} \sum_{i=0}^{\infty}\left(\begin{array}{c}
(k / \beta) \\
i
\end{array}\right)(-1)^{(k / \beta-i)} \Gamma\left(\frac{i}{\alpha}+1,1\right) .
$$

and

The incomplete $r$ th moment expression for PGW ( $T>t$ and $T<t$ ) is

$$
\mathbb{E}\left(T^{k} \mid T>t\right)=\frac{e}{\lambda^{(k / \beta)}} \sum_{i=0}^{\infty}\left(\begin{array}{c}
(k / \beta) \\
i
\end{array}\right)(-1)^{(k / \beta-i)}\left[\Gamma\left(\frac{i}{\alpha}+1,1\right)-\Gamma\left(\frac{i}{\alpha}+1, \emptyset\right)\right]
$$

\section{Alternate Expressions for the rth Moments of the PGW (or DAL) Model}

The $r$ th moment expression for the PGW model becomes

$$
\mathbb{E}\left(T^{k}\right)=\frac{e}{\lambda^{(k / \beta)}} \int_{1}^{\infty}(-1)^{(k / \beta)}\left(1-\varpi^{1 / \alpha}\right)^{(k / \beta)} \exp \{-\emptyset\} d \Phi,
$$

which, after using binomial expansion, results in

$$
\mathbb{E}\left(T^{k}\right)=\frac{e}{\lambda^{(k / \beta)}}(-1)^{(k / \beta)} \sum_{i=0}^{\infty} a_{i}(k / \beta) \Gamma\left(\frac{i}{\alpha}+1,1\right)
$$

The alternate $r$ th incomplete moment expressions for PGW $(T>t$ and $T<t)$ are

$$
\mathbb{E}\left(T^{k} \mid T>t\right)=\frac{e}{\lambda^{(k / \beta)}}(-1)^{(k / \beta)} \sum_{i=0}^{\infty} a_{i}(k / \beta) \int_{\omega}^{\infty} \omega^{(i / \alpha)} \exp \{-\omega\} d \omega=\frac{e}{\lambda^{(k / \beta)}}(-1)^{(k / \beta)} \sum_{i=0}^{\infty} a_{i}(k / \beta)\left[\Gamma\left(\frac{i}{\alpha}+1,1\right)-\Gamma\left(\frac{i}{\alpha}+1, \omega\right)\right]
$$

and

$$
\mathbb{E}\left(T^{k} \mid T<t\right)=\frac{e}{\lambda^{(k / \beta)}}(-1)^{(k / \beta)} \sum_{i=0}^{\infty} a_{i}(k / \beta) \int_{\omega}^{\infty} \omega^{(i / \alpha)} \exp \{-\omega\} d \omega=\frac{e}{\lambda^{(k / \beta)}}(-1)^{(k / \beta)} \sum_{i=0}^{\infty} a_{i}(k / \beta)\left[\Gamma\left(\frac{i}{\alpha}+1,1\right)-\Gamma\left(\frac{i}{\alpha}+1, \omega\right)\right]
$$

\section{Empirical Investigation}

In this section, we empirically show the comparison among three models, described in the paper, which are NH, DAL, and PGW distributions. Two real-life datasets are used to compare and illustrate the potentiality of $\mathrm{NH}, \mathrm{DAL}$, and PGW models. The datasets are given as follows.

Dataset 1. (failure time data). The first real dataset is taken from [99] which represents the 50 observations of load, haul, dump machine- $C$ failure time. The data are as follows: 110 , $13,72,4,45,56,19,57,36,90,19,7,2,118,44,8,277,4,8,10$,
$79,103,6,18,147,96,22,3,24,3,9,99,82,121,54,79,99,18$, $5,21,1,3,5,1,59,22,17,35,35$, and 29 .

Dataset 2. (failure time data). The second dataset is taken from [100] which represents the failure time of $20 \mathrm{com}-$ ponents. The data are as follows: $0.072,4.763,8.663,12.089$, $0.477,5.284,9.511,13.036,1.592,7.709,10.636,13.949$, $2.475,7.867,10.729,16.169,3.597,8.661,11.501$, and 19.809 .

The AdequacyModel package is used in R-Statistical Computing Environment to compute maximum likelihood estimates (MLEs) and the standard errors (SEs) of the estimates of the proposed and other competitive models. The 
TABLE 4: MLEs and their SEs (in parentheses) for dataset 1.

\begin{tabular}{lcccc}
\hline Distribution & $\alpha$ & $\lambda$ & $\beta$ & $\gamma$ \\
\hline \multirow{2}{*}{ NH } & 0.6711 & 0.0444 & - & - \\
& $(0.1907)$ & $(0.0248)$ & 0.8760 & - \\
\hline \multirow{2}{*}{ DAL } & 0.9673 & 0.0392 & $(0.2419)$ & - \\
\hline \multirow{2}{*}{ PGW } & $(0.8683)$ & $(0.0264)$ & 0.8927 & $(0.2427)$ \\
\end{tabular}

TABLE 5: MLEs and their standard errors (in parentheses) for dataset 2.

\begin{tabular}{|c|c|c|c|c|}
\hline Distribution & $\alpha$ & $\lambda$ & $\beta$ & $\gamma$ \\
\hline $\mathrm{NH}$ & $\begin{array}{c}17.6456 \\
(18.8952)\end{array}$ & $\begin{array}{c}0.0044 \\
(0.0048)\end{array}$ & - & - \\
\hline DAL & $\begin{array}{c}9.3155 \\
(11.5537)\end{array}$ & $\begin{array}{c}0.0065 \\
(0.0077)\end{array}$ & $\begin{array}{c}1.1063 \\
(0.2211) \\
\end{array}$ & $\begin{array}{l}- \\
-\end{array}$ \\
\hline PGW & $\begin{array}{l}- \\
-\end{array}$ & $\begin{array}{c}68.1396 \\
(97.9441)\end{array}$ & $\begin{array}{c}1.1391 \\
(0.2167)\end{array}$ & $\begin{array}{c}0.1428 \\
(0.2065)\end{array}$ \\
\hline
\end{tabular}

TABle 6: The statistics AIC, BIC, HQIC, AD, CvM, and KS and $p$ value for dataset 1.

\begin{tabular}{lccccccccc}
\hline Distribution & $-\hat{\ell}$ & AIC & BIC & CAIC & HQIC & AD & CvM & KS & KS $p$ value \\
\hline NH & 240.5352 & 485.0704 & 488.8944 & 485.3257 & 486.5266 & 0.4601 & 0.0681 & 0.0855 & 0.8580 \\
DAL & 240.4329 & 486.8659 & 492.6020 & 487.3876 & 489.0502 & 0.4389 & 0.0649 & 0.0790 & 0.9140 \\
PGW & 240.4344 & 486.8689 & 492.6050 & 487.3906 & 489.0532 & 0.4411 & 0.0652 & 0.0802 & 0.9050 \\
\hline
\end{tabular}

TABle 7: The statistics AIC, BIC, HQIC, AD, CvM, and KS and $p$ value for dataset 2.

\begin{tabular}{lccccccccc}
\hline Distribution & $-\hat{\ell}$ & AIC & BIC & CAIC & HQIC & AD & CvM & KS & KS $p$ value \\
\hline NH & 59.9218 & 123.8435 & 125.8350 & 124.5494 & 124.2323 & 0.4490 & 0.0785 & 0.2056 & 0.3211 \\
DAL & 59.8936 & 125.7872 & 128.7744 & 127.2872 & 126.3703 & 0.4282 & 0.0749 & 0.1822 & 0.4659 \\
PGW & 59.9544 & 125.9089 & 128.8961 & 127.4089 & 126.4920 & 0.4282 & 0.0749 & 0.1795 & 0.4848 \\
\hline
\end{tabular}

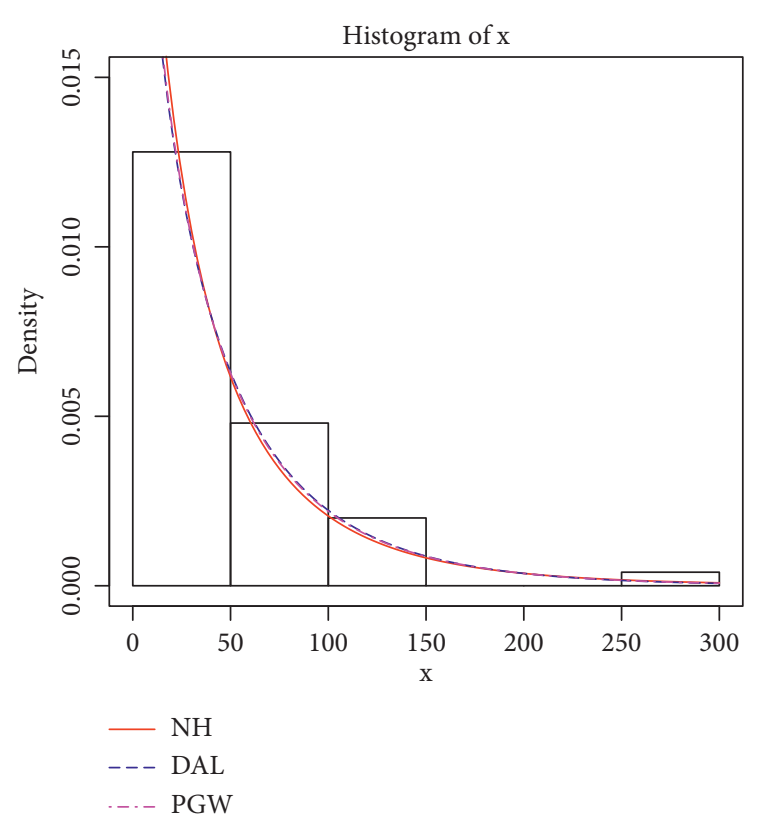

(a)

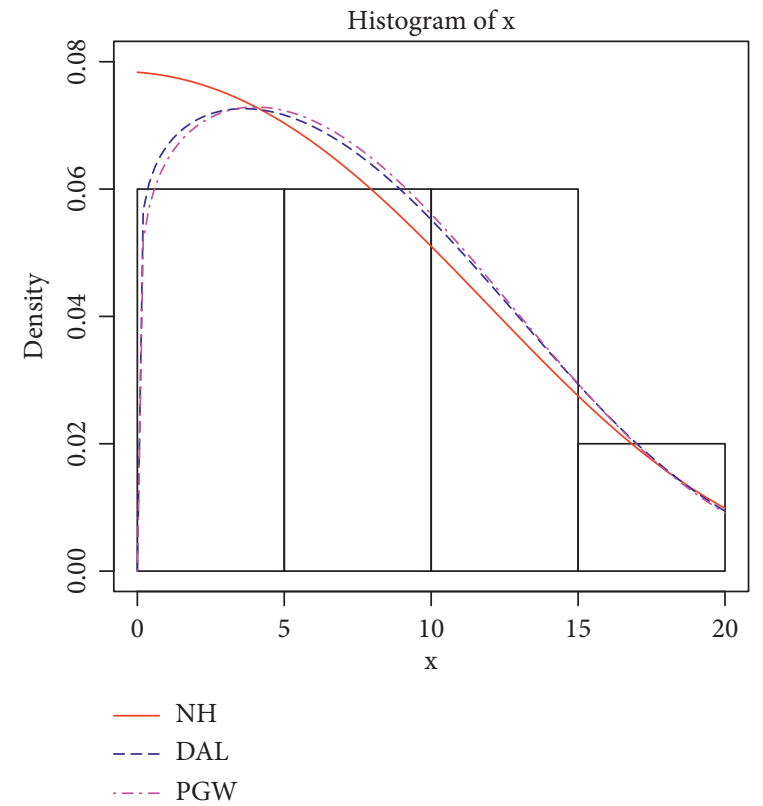

(b)

Figure 1: Plots of the estimated pdf of NH, DAL, and PGW models for datasets (a) 1 and (b) 2. 


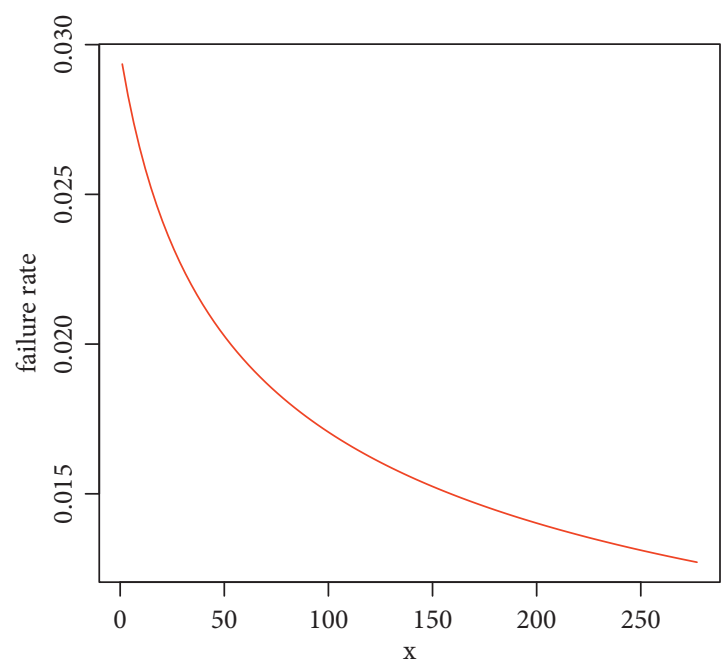

$-\mathrm{NH}$

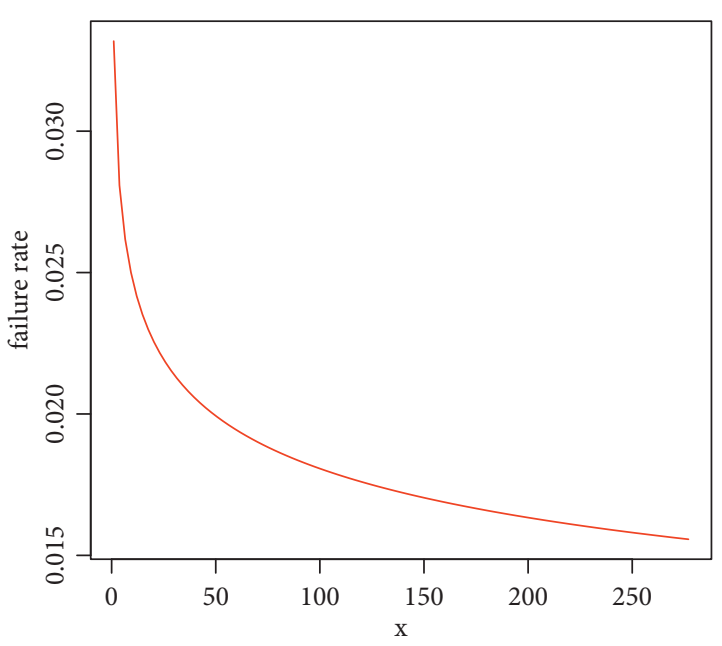

- DAL

(a)

(b)

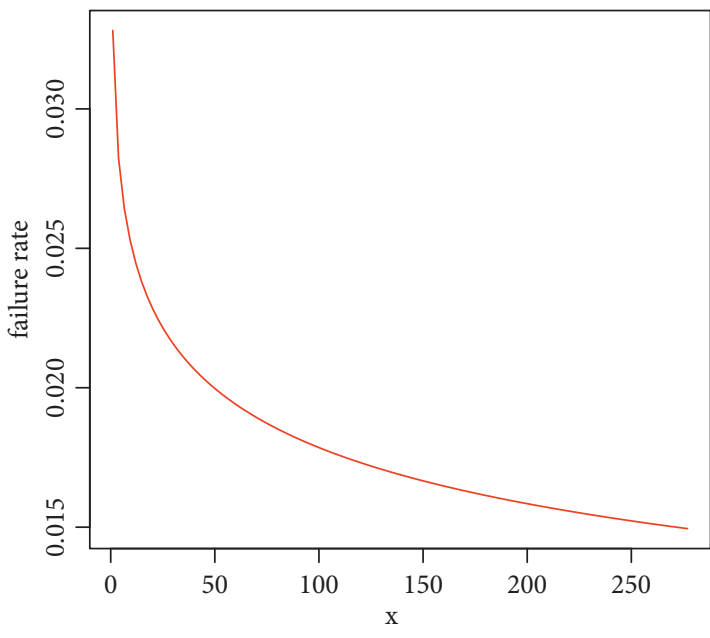

— PGW

(c)

Figure 2: Plots of the estimated hazard rate of (a) NH, (b) DAL, and (c) PGW models for dataset 1.

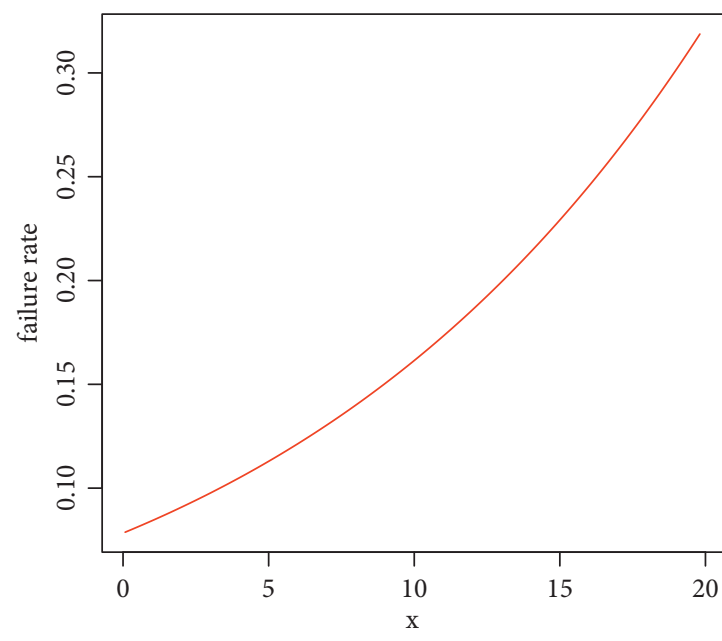

$-\mathrm{NH}$

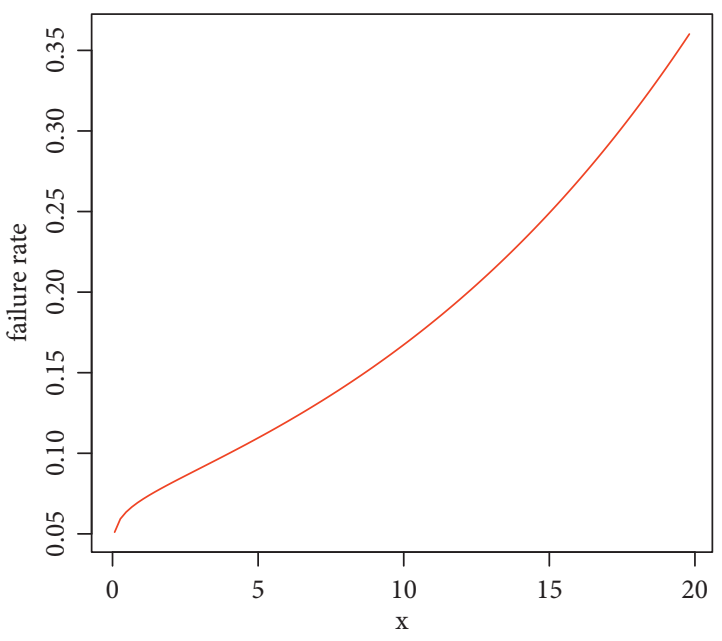

DAL

(a)

(b)

Figure 3: Continued. 


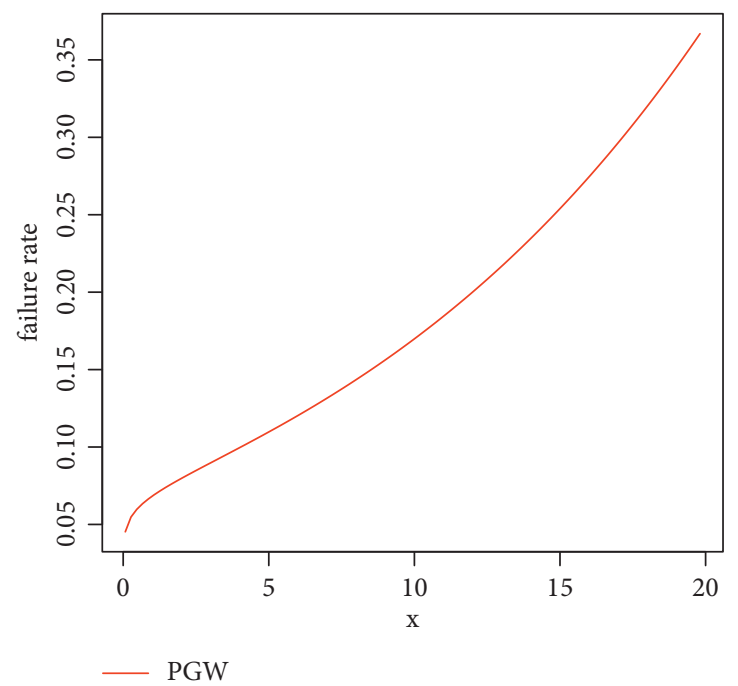

(c)

FIGURE 3: Plots of the estimated hazard rate of (a) NH, (b) DAL, and (c) PGW models for dataset 2.

log-likelihood function is evaluated at the MLEs $(\widehat{\ell})$. Some well-established goodness-of-fit (GoF) statistics such as Akaike information criterion (AIC), Bayesian information criterion (BIC), consistent Akaike's information criterion (CAIC), Hannan-Quinn information criterion (HQIC), Anderson-Darling (AD), Cramér-von Mises (CvM), and Kolmogorov-Smirnov (KS) are used for model comparison purposes. The low values of GoFs and high KS $p$ values indicate good fits.

Tables 4 and 5 list the MLEs and their SEs, and Tables 6 and 7 report the values of the GoFs. For dataset 1, the model $\mathrm{NH}$ is better in performance as compared to DAL and PGW while considering GoFs AIC, BIC, CAIC, and HQIC. The model DAL is better as compared to $\mathrm{NH}$ and PGW if GoFs $\mathrm{AD}, \mathrm{CvM}$, and $\mathrm{KS}$ are considered. For dataset 2, the model $\mathrm{NH}$ is better in performance as compared to DAL and PGW while considering GoFs AIC, BIC, CAIC, and HQIC. The model PGW is better as compared to NH and DAL if GoFs $\mathrm{AD}, \mathrm{CvM}$, and $\mathrm{KS}$ are considered. Figures 1-3 show the estimated pdf and hazard rate of $\mathrm{NH}, \mathrm{DAL}$, and PGW, which support our results in Tables 6 and 7 .

\section{Concluding Remarks}

In this article, we dealt the following: (i) we investigated an unbiased and robust investigation of the development of the three interrelated models and gave due credit to the authors who actually deserve, (ii) we presented an updated review of the literature on NH, PGW, and DAL extended models and their related G-classes, (iii) we pointed out mistakes (or typos) in the moments' section of the NH paper published in the year 2011, and (iv) we provided corrected and extended moments and moment-generating expressions, and lastly, an empirical investigation was carried out where the three models were compared on two datasets.

\section{Data Availability}

The data used to support the findings of this study are included within the article.

\section{Conflicts of Interest}

The authors declare that they have no conflicts of interest.

\section{References}

[1] S. Nadarajah and F. Haghighi, "An extension of the exponential distribution," Statistics, vol. 45 , no. 6, pp. 543-558, 2011.

[2] V. Bagdonavičios and M. Nikulin, Accelerated Life Models: Modeling and Statistical Analysis, Chapman and Hall/CRC, London, UK, 2002.

[3] M. Nikulin and F. Haghighi, "A chi-squared test for the generalized power Weibull family for the head-and-neck cancer censored data," Journal of Mathematical Sciences, vol. 133, no. 3, pp. 1333-1341, 2006.

[4] T. Dimitrakopoulou, K. Adamidis, and S. Loukas, "A lifetime distribution with an upside-down bathtub-shaped hazard function," IEEE Transactions on Reliability, vol. 56, no. 2, pp. 308-311, 2007.

[5] S. Nadarajah, "On the moments of the modified Weibull distribution," Reliability Engineering \& System Safety, vol. 90, no. 1, pp. 114-117, 2005.

[6] M. Xie, Y. Tang, and T. N. Goh, "A modified Weibull extension with bathtub-shaped failure rate function," Reliability Engineering \& System Safety, vol. 76, no. 3, pp. 279-285, 2002.

[7] S. Nadarajah and S. Kotz, "On some recent modifications of Weibull distribution," IEEE Transactions on Reliability, vol. 54, no. 4, pp. 561-562, 2005.

[8] M. R. Gurvich, A. T. Dibenedetto, and S. V. Ranade, "A new statistical distribution for characterizing the random 
strength of brittle materials," Journal of Materials Science, vol. 32, no. 10, pp. 2559-2564, 1997.

[9] S. Nadarajah and S. Kotz, "The two-parameter bathtubshaped lifetime distribution," Quality and Reliability Engineering International, vol. 23, no. 2, pp. 279-280, 2007.

[10] J.-W. Wu, H.-L. Lu, C.-H. Chen, and C.-H. Wu, "Statistical inference about the shape parameter of the new two-parameter bathtub-shaped lifetime distribution," Quality and Reliability Engineering International, vol. 20, no. 6, pp. 607-616, 2004.

[11] Z. Chen, "A new two-parameter lifetime distribution with bathtub shape or increasing failure rate function," Statistics \& Probability Letters, vol. 49, no. 2, pp. 155-161, 2000.

[12] H. A. Bidram, M. Roman, and V. G. Cancho, "The complementary exponential geometric distribution: model, properties, and a comparison with its counterpart. Computational Statistics and Data Analysis 55:2516-2524"," Computational Statistics \& Data Analysis, vol. 74, p. 180, 2011.

[13] C.-S. Lee and H.-J. Tsai, "A note on the generalized linear exponential distribution," Statistics \& Probability Letters, vol. 124, pp. 49-54, 2017.

[14] M. A. W. Mahmoud and F. M. A. Alam, "The generalized linear exponential distribution," Statistics \& Probability Letters, vol. 80, no. 11-12, pp. 1005-1014, 2010.

[15] I. E. Okorie and A. C. Akpanta, "A note on the transmuted generalized inverted exponential distribution with application to reliability data," Thailand Statistics, vol. 17, pp. 118-124, 2019.

[16] I. Elbatal, "Transmuted generalized inverted exponential distribution," Stochastics and Quality Control, vol. 28, pp. 125-133, 2013.

[17] M. S. Khan, "Transmuted generalized inverted exponential distribution with application to reliability data," Thailand Statistics, vol. 16, pp. 14-25, 2018a.

[18] M. V. Aarset, "How to identify a bathtub hazard rate," IEEE Transactions on Reliability, vol. 36, no. 1, pp. 106-108, 1987.

[19] S. Nadarajah and I. E. Okorie, "A note on a new member from the T-X family of distributions: the gumbel-burr XII distribution and its properties," Sankhya A, vol. 82, no. 1, pp. 257-259, 2020.

[20] P. Osatohanmwen, F. O. Oyegue, and S. M. Ogbonmwan, “A new Member from the $\mathrm{T}-\mathrm{X}$ family of distributions: the Gumbel-Burr XII distribution and its properties," Sankhya A, vol. 81, no. 2, pp. 298-322, 2019.

[21] S. Nadarajah and Y. Zhang, "A note on the transmuted inverse Weibull distribution," Thailand Statistics, vol. 18, pp. 90-94, 2020.

[22] M. S. Khan, R. King, and I. L. Hudson, "Characteristics of the transmuted inverse Weibull distribution," ANZIAM Journal, vol. 55, pp. C197-C217, 2014.

[23] S. Nadarajah and S. Chan, "On moments of the unit Lindley distribution," Journal of Applied Statistics, vol. 47, no. 5, pp. 947-949, 2020.

[24] J. Mazucheli, A. F. B. Menezes, and S. Chakraborty, "On the one parameter unit-Lindley distribution and its associated regression model for proportion data," Journal of Applied Statistics, vol. 46, no. 4, pp. 700-714, 2019.

[25] L. J. Bain, "Analysis for the linear failure-rate life-testing distribution," Technometrics, vol. 16, no. 4, pp. 551-559, 1974.

[26] R. D. Gupta and D. Kundu, "Generalized exponential distributions," Australian New Zealand Journal of Statistics, vol. 41, no. 2, pp. 173-188, 1999.
[27] S. M. Mirhossaini and A. Dolati, "On a new generalization of the exponential distribution," Journal of Mathematical Extension, vol. 3, pp. 27-42, 2008.

[28] S. Çelebioğlu, "On the extension of the exponential and Weibull distributions," SDU Journal of Science, vol. 5, pp. 137-146, 2010.

[29] A. Olapade, "On extended generalized exponential distribution," British Journal of Mathematics \& Computer Science, vol. 4, no. 9, pp. 1280-1289, 2014.

[30] Y. M. Gómez, H. Bolfarine, and H. W. Gómez, "A new extension of the exponential distribution," Revista Colombiana de Estadística, vol. 37, no. 1, pp. 25-34, 2014.

[31] M. Nikulin and F. Haghighi, "A chi-squared test for the generalized power Weibull family for the head-and-neck cancer censored data," Journal of Mathematical Sciences, vol. 133, no. 3, pp. 1333-1341, 2006.

[32] M. Nikulin and F. Haghighi, "On the power generalized Weibull family: model for cancer censored data," Metron, vol. 67, pp. 75-86, 2009.

[33] F. A. Peña-ramírez, R. R. Guerra, G. M. Cordeiro, and P. R. D. Marinho, "The exponentiated power generalized Weibull: properties and applications," Anais da Academia Brasileira de Ciências, vol. 90, no. 3, pp. 2553-2577, 2018.

[34] A. Alzaatreh, C. Lee, and F. Famoye, "A new method for generating families of continuous distributions," Metron, vol. 71, no. 1, pp. 63-79, 2013.

[35] B. Gompertz, "On the nature of the function expressive of the law of human mortality and on the new mode of determining the value of life contingencies," Philosophical Transactions of the Royal Society, vol. A115, pp. 513-580, 1825.

[36] D. Kumar, S. Dey, and S. Nadarajah, "Extended exponential distribution based on order statistics," Communications in Statistics-Theory and Methods, vol. 46, no. 18, pp. 9166-9184, 2017.

[37] S. M. T. K. MirMostafaee, A. Asgharzadeh, and A. Fallah, "Record values from $\mathrm{NH}$ distribution and associated inference," Metron, vol. 74, no. 1, pp. 37-59, 2016.

[38] M. A. Selim, "Estimation and prediction for NadarajahHaghighi distribution based on record values," Pakistan Journal of Statistics, vol. 34, pp. 77-90, 2018.

[39] M. J. S. K. Khan, A. Sharma, and A. Sharma, "Shannon entropy and characterization of Nadarajah and Haghighi distribution based on generalized order statistics," Journal of Statistics: Advances in Theory and Applications, vol. 19, no. 1, pp. 43-69, 2018.

[40] M. Sana and M. Faizan, "Bayesian estimation for NadarajahHaghighi distribution based on upper record values," Pakistan Journal of Statistics and Operation Research, vol. 15, pp. 217-230, 2019.

[41] F. Haghighi, "Optimal design of accelerated life tests for an extension of the exponential distribution," Reliability Engineering \& System Safety, vol. 131, pp. 251-256, 2014.

[42] F. Haghighi, "Simple step-stress model for an extension of the exponential distribution with type-I censoring," International Journal of Quality \& Reliability Management, vol. 32, no. 8, pp. 906-920, 2015.

[43] M. M. M. El-Din, S. E. Abu-Youssef, N. S. A. Ali, and A. M. A. El-Raheem, "Parametric inference on step-stress accelerated life testing for the extension of exponential distribution under progressive type-II censoring," Communications for Statistical Applications and Methods, vol. 23, no. 4, pp. 269-285, 2016.

[44] M. M. Mohie El-Din, S. E. Abu-Youssef, N. S. A. Ali, and A. M. Abd El-Raheem, "Classical and Bayesian inference on 
progressive-stress accelerated life testing for the extension of the exponential distribution under progressive type-II censoring," Quality and Reliability Engineering International, vol. 33, no. 8, pp. 2483-2496, 2017.

[45] S. K. Singh, U. Singh, M. Kumar, and P. K. Vishwakarma, "Classical and Bayesian inference for an extension of the exponential distribution under progressive type-II censored data with binomial removals," Journal of Statistics Applications \& Probability Letters, vol. 1, no. 3, pp. 75-86, 2014.

[46] A. M. Abd El-Raheem, "Optimal design of multiple constantstress accelerated life testing for the extension of the exponential distribution under type-II censoring," Journal of Computational and Applied Mathematics, vol. 382, Article ID 113094, 2021.

[47] M. Kumar, S. K. Singh, and U. Singh, "Extension of exponential count model and its application to emissions of beta particles from a nuclear reaction," Journal of Advanced Statistics, vol. 1, pp. 136-145, 2016.

[48] S. Ali, M. Shafqat, I. Shah, and S. Dey, "Bivariate discrete Nadarajah and Haghighi distribution: properties and different methods of estimation," Filomat, vol. 33, no. 17, pp. 5589-5610, 2019.

[49] S. Nasiru, A. G. Abubakari, and J. Abonongo, "Quantile generated Nadarajah-Haghighi family of distributions," Annals of Data Science, 2020a.

[50] M. A. Aljarrah, C. Lee, and F. Famoye, "On generating T-X family of distributions using quantile functions," Journal of Statistical Distributions and Applications, vol. 1, 2014.

[51] S. Nasiru, A. G. Abubakari, and J. Abonongo, "Unit Nadarajah-Haghighi generated family of distributions: properties and applications," Sankhyã Series A: The Indian Journal of Statistics, vol. 115, 2020.

[52] A. Alzaatreh, M. A. Aljarrah, M. Smithson et al., "Truncated family of distributions with applications to time and cost to start a business," Methodology and Computing in Applied Probability, vol. 23, 2020.

[53] S. K. Singh, U. Singh, and A. S. Yadav, "Reliability estimation and prediction for extension of exponential distribution using informative and non-informative priors," International Journal of System Assurance Engineering and Management, vol. 6, no. 4, pp. 466-478, 2015.

[54] S. Dey, C. Zhang, A. Asgharzadeh, and M. Ghorbannezhad, "Comparisons of methods of estimation for the NH distribution," Annals of Data Science, vol. 4, no. 4, pp. 441-455, 2017.

[55] M. Minic, "Estimation of parameters of Nadarajah-Haghighi extension of the exponential distribution using perfect and imperfect ranked set sample," Yugoslav Journal of Operations Research, vol. 30, no. 2, pp. 177-198, 2020.

[56] M. A. El-Damcese, "Studies on properties and estimation problems for modified extension of exponential distribution," International Journal of Computer Applications, vol. 125, no. 4, pp. 21-28, 2015.

[57] M. N. Khan, A. Saboor, G. M. Cordeiro, M. Nazir, and R. R. Pescim, "A weighted Nadarajah-Haghighi distribution," UPB Scientific Bulletin, Series A: Applied Mathematics and Physics, vol. 80, pp. 133-140, 2018.

[58] F. A. Peña-Ramírez, R. R. Guerra, and G. M. Cordeiro, "The nadarajah-haghighi Lindley distribution," Anais da Academia Brasileira de Ciências, vol. 91, Article ID e20170856, 2019.

[59] A. J. Lemonte, "A new exponential-type distribution with constant, decreasing, increasing, upside-down bathtub and bathtub-shaped failure rate function," Computational Statistics \& Data Analysis, vol. 62, pp. 149-170, 2013.

[60] R. C. Gupta, P. L. Gupta, and R. D. Gupta, "Modeling failure time data by Lehman alternatives," Communications in Statistics - Theory and Methods, vol. 27, no. 4, pp. 887-904, 1998.

[61] D. Sira KA, G. O. Orwa, and O. Ngesa, "Exponentiated Nadarajah Haghighi Poisson distribution," International Journal of Statistics and Probability, vol. 8, no. 5, pp. 34-48, 2019.

[62] A. Saboor, M. N. Khan, G. M. Cordeiro, I. Elbatal, and R. R. Pescim, "The beta exponentiated Nadarajah-Haghighi distribution: theory, regression model and application," Mathematica Slovaca, vol. 69, no. 4, pp. 939-952, 2019.

[63] Z. A. Alhussain and E. A. Ahmed, "Estimation of exponentiated Nadarajah-Haghighi distribution under progressively type-II censored sample with application to bladder cancer data," Indian Journal of Pure and Applied Mathematics, vol. 51, no. 2, pp. 631-657, 2020.

[64] M. H. Tahir, G. M. Cordeiro, S. Ali, S. Dey, and A. Manzoor, "The inverted Nadarajah-Haghighi distribution: estimation methods and applications," Journal of Statistical Computation and Simulation, vol. 88, no. 14, pp. 2775-2798, 2018.

[65] G. Raffiq, I. S. Dar, M. A. U. Haq, and E. Ramos, "The marshall-olkin inverted nadarajah-haghighi distribution: estimation and applications," Annals of Data Science, vol. 79, 2020.

[66] G. S. Mudholkar and D. K. Srivastava, "Exponentiated Weibull family for analyzing bathtub failure-rate data," IEEE Transactions on Reliability, vol. 42, no. 2, pp. 299-302, 1993.

[67] G. S. Mudholkar, D. K. Srivastava, and M. Freimer, "The exponentiated Weibull family: a reanalysis of the bus-motorfailure data," Technometrics, vol. 37, no. 4, pp. 436-445, 1995.

[68] V. Voinov, N. Pya, N. Shapakov, and Y. Voinov, "Goodnessof-fit tests for the power-generalized Weibull probability distribution," Communications in Statistics - Simulation and Computation, vol. 42, no. 5, pp. 1003-1012, 2013.

[69] D. Kumar and S. Dey, "Power generalized Weibull distribution based on order Statistics," Journal of Statistical Research, vol. 51, no. 1, pp. 61-78, 2017.

[70] D. Kumar and N. Jain, "Power generalized Weibull based on generalized order statistics," J. Data Sci.vol. 16, pp. 621-646, 2018.

[71] R. Pandey and N. Kumari, "Bayesian analysis of power generalized Weibull distribution," International Journal of Applied and Computational Mathematics, vol. 4, 2018.

[72] M. A. Sabry, H. Z. Muhammed, A. Nabih, and M. Shaaban, "Parameter estimation for the power generalized Weibull distribution based on one- and two-stage ranked set sampling designs," Journal of Applied Probability and Statistics, vol. 8, pp. 113-128, 2019.

[73] E. M. Almetwaly and H. M. Almongy, "Estimation of the generalized power Weibull distribution parameters using progressive censoring schemes," International Journal of Statistics and Probability, vol. 7, pp. 51-61, 2018.

[74] M. M. M. El-Din, A. M. A. El-Raheem, and O. S. A. ElAzeem, "On step-stress accelerated life testing for power generalized Weibull distribution under progressive type-II censoring," Annals of Data Science, vol. 8, 2020.

[75] M. Jones, A. Noufaily, and K. Burke, "A bivariate power generalized Weibull distribution: a flexible parametric model for survival analysis," Statistical Methods in Medical Research, vol. 29, no. 8, pp. 2295-2306, 2019. 
[76] K. Zografos and N. Balakrishnan, "On families of beta- and generalized gamma-generated distributions and associated inference," Statistical Methodology, vol. 6, no. 4, pp. 344-362, 2009.

[77] W. T. Shaw and I. R. Buckley, "The alchemy of probability distributions: beyond gram-charlier expansions and a skewkurtotic normal distribution from a rank transmutation map," 2007, https://arxiv.org/abs/0901.0434.

[78] G. M. Cordeiro, E. M. M. Ortega, and D. C. C. da-Cunha, "The exponentiated generalized class of distributions," Journal of Data Science, vol. 11, pp. 1-27, 2013.

[79] A. Marshall and I. Olkin, "A new method for adding a parameter to a family of distributions with application to the exponential and Weibull families," Biometrika, vol. 84, no. 3, pp. 641-652, 1997.

[80] Y. Sangsanit and W. Bodhisuwan, "The Topp-Leone generator of distributions: properties and inferences," Songklanakarin Journal of Science and Technology, vol. 38, pp. 537-548, 2016.

[81] F. S. Gomes-Silva, A. Percontini, E. d. Brito, M. W. Ramos, R. Venâncio, and G. M. Cordeiro, "The odd lindley-G family of distributions," Austrian Journal of Statistics, vol. 46, no. 1, pp. 65-87, 2017.

[82] M. Bourguignon, R. B. Silva, and G. M. Cordeiro, "The Weibull-G family of probability distributions," Journal of Data Science, vol. 12, pp. 53-68, 2014.

[83] G. M. Cordeiro and M. de Castro, "A new family of generalized distributions," Journal of Statistical Computation and Simulation, vol. 81, no. 7, pp. 883-898, 2011.

[84] N. Eugene, C. Lee, and F. Famoye, "Beta-normal distribution and its applications," Communications in Statistics - Theory and Methods, vol. 31, no. 4, pp. 497-512, 2002.

[85] G. M. Cordeiro, M. Alizadeh, and P. R. Diniz Marinho, "The type I half-logistic family of distributions," Journal of Statistical Computation and Simulation, vol. 86, no. 4, pp. 707-728, 2016.

[86] M. Alizadeh, M. Afshari, B. Hosseini, and T. G. Ramires, "Extended exp-G family of distributions: properties, applications and simulation," Communications in Statistics Simulation and Computation, vol. 49, no. 7, pp. 1730-1745, 2020.

[87] H. M. Yousof, A. Z. Afify, G. G. Hamedani, and G. Aryal, "The Burr X generator of distributions for lifetime data," Journal of Statistical Theory and Applications, vol. 16, no. 3, pp. 288-305, 2017.

[88] A. Mahdavi and D. Kundu, "A new method for generating distributions with an application to exponential distribution," Communications in Statistics-Theory and Methods, vol. 46, no. 13, pp. 6543-6557, 2017.

[89] J. U. Gleaton and J. D. Lynch, "Properties of generalized loglogistic families of lifetime distributions," Journal of Probability and Statistics, vol. 4, pp. 51-64, 2006.

[90] M. H. Tahir, G. M. Cordeiro, A. Alzaatreh, M. Mansoor, and M. Zubair, "The logistic-X family of distributions and its applications," Communications in Statistics - Theory and Methods, vol. 45, no. 24, pp. 7326-7349, 2016.

[91] Z. Ahmad, M. Elgarhy, and G. G. Hamedani, "A new Weibull-X family of distributions: properties, characterizations and applications," Journal of Statistical Distributions and Applications, vol. 5, 2018.

[92] A. D. C. Nascimento, K. F. Silva, G. M. Cordeiro, M. Alizadeh, H. M. Yousof, and G. G. Hamedani, "The odd Nadarajah-Haghighi family of distributions: properties and applications," Studia Scientiarum Mathematicarum Hungarica, vol. 56, no. 2, pp. 185-210, 2019.

[93] H. Reyad, M. A. Selim, and S. Othman, "The Nadarajah Haghighi topp leone-G family of distributions with mathematical properties and applications," Pakistan Journal of Statistics and Operation Research, vol. 15, no. 4, pp. 849-866, 2019.

[94] Z. Ahmad, M. Elgarhy, G. G. Hamedani, and N. S. Butt, "Odd generalized N-H generated family of distributions with application to exponential model," Pakistan Journal of Statistics and Operation Research, vol. 16, pp. 53-71, 2020.

[95] S. Nasiru and A. G. Abubakari, "Complementary generalized power Weibull power series family of distributions: estimation and application," Eurasian Bulletin of Mathematics, vol. 3, pp. 20-37, 2020.

[96] M. A. Aldahlan, F. Jamal, C. Chesneau, I. Elbatal, and M. Elgarhy, "Exponentiated power generalized Weibull power series family of distributions: properties, estimation and applications," Plos One, vol. 15, 2020.

[97] G. M. Cordeiro and M. G. de Andrade, "Transformed generalized linear models," Journal of Statistical Planning and Inference, vol. 139, no. 9, pp. 2970-2987, 2009.

[98] G. M. Cordeiro and M. G. Andrade, "Transformed symmetric models," Statistical Modelling, vol. 11, no. 4, pp. 371-388, 2011.

[99] U. Kumar, B. Klefsjö, and S. Granholm, "Reliability investigation for a fleet of load haul dump machines in a Swedish mine," Reliability Engineering \& System Safety, vol. 26, no. 4, pp. 341-361, 1989.

[100] D. N. P. Murthy, M. Xie, and R. Jiang, Weibull Models, Wiley, New York, NY, USA, 2004. 